\title{
Editorial
}

\section{Advances on Multivalued Operators and Related Fixed Point Problems}

\author{
Chi-Ming Chen, ${ }^{1}$ Erdal Karapınar, ${ }^{2,3}$ Wei-Shih Du, ${ }^{4}$ \\ Hassen Aydi, ${ }^{5}$ and Salvador Romaguera ${ }^{6}$ \\ ${ }^{1}$ Department of Applied Mathematics, National Hsinchu University of Education, Taiwan \\ ${ }^{2}$ Department of Mathematics, Atilim University, Incek, 06836 Ankara, Turkey \\ ${ }^{3}$ Nonlinear Analysis and Applied Mathematics Research Group (NAAM), King Abdulaziz University, Jeddah, Saudi Arabia \\ ${ }^{4}$ Department of Mathematics, National Kaohsiung Normal University, Kaohsiung 824, Taiwan \\ ${ }^{5}$ Department of Mathematics, College of Education of Jubai, Dammam University, Dammam, Saudi Arabia \\ ${ }^{6}$ Instituto Universitario de Matemática Puray Aplicada, Universitat Politècnica de València, Camí de Vera s/n, 46022 Valencia, Spain
}

Correspondence should be addressed to Chi-Ming Chen; ming@mail.nhcue.edu.tw

Received 25 February 2014; Accepted 25 February 2014; Published 7 April 2014

Copyright (C) 2014 Chi-Ming Chen et al. This is an open access article distributed under the Creative Commons Attribution License, which permits unrestricted use, distribution, and reproduction in any medium, provided the original work is properly cited.

It is a dispensable fact that the fixed point theory has a key role in solving various problems in nonlinear sciences. Most of the time, real life problems can be easily converted to the problem of existence and uniqueness of a fixed point for maps. Consequently, fixed point techniques have been used quite extensively in different quantitative sciences such as economics, computer science, engineering, biology, chemistry, and physics. Regarding its wide application potential, fixed point theory has been improved by a number of authors to investigate new techniques and approached to determine the existence and uniqueness of a fixed point of various operators in distinct abstract spaces to solve practical problems arising in natural sciences. In particular, we collect fixed point theory papers devoted to the existence of multivalued operators.

The aim of this special issue is to bring together the qualified outstanding results in fixed point theory and discuss their possible applications not only in the branches of mathematics but also in some other quantitative sciences.

The most difficult part of editing this special issue was to choose from the 61 high-quality papers. Among them, we have chosen 43 high-quality peer-reviewed papers from different aspects of theory and applications of fixed points. These papers, published in this special issue, have novelty and contain some interesting, creative, and prominent ideas. We do believe that all the papers published in this special issue will active and prompt further scientific activities in the field of fixed point theory and its applications.

\section{Acknowledgment}

We would like to express our deepest gratitude to a lot of reviewers, whose professional comments guaranteed the high quality of the selected papers.

Chi-Ming Chen

Erdal Karapinar

Wei-Shih Du

Hassen Aydi

Salvador Romaguera 


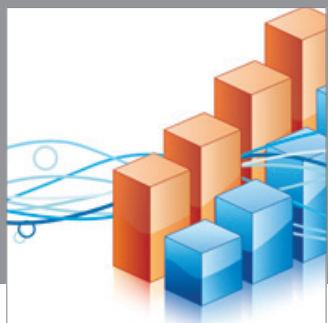

Advances in

Operations Research

mansans

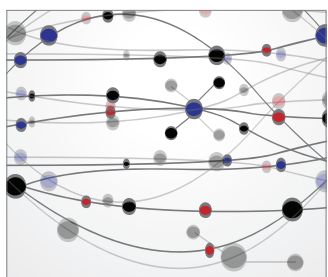

The Scientific World Journal
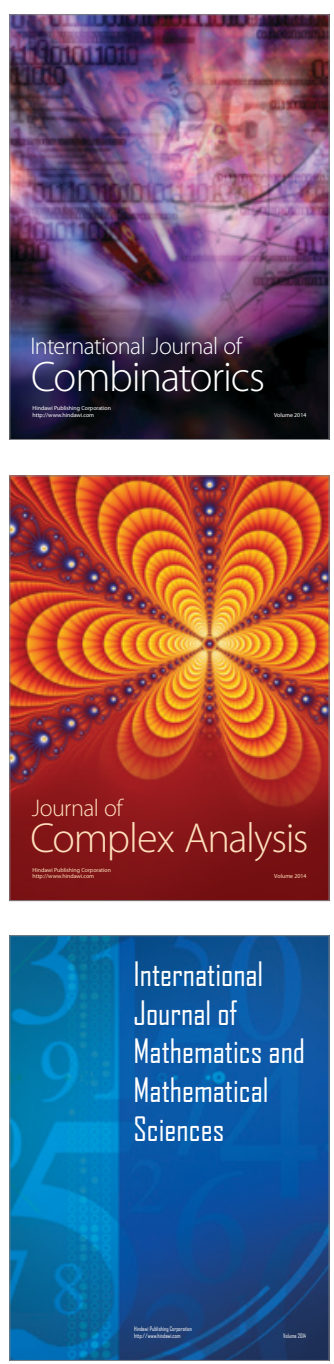
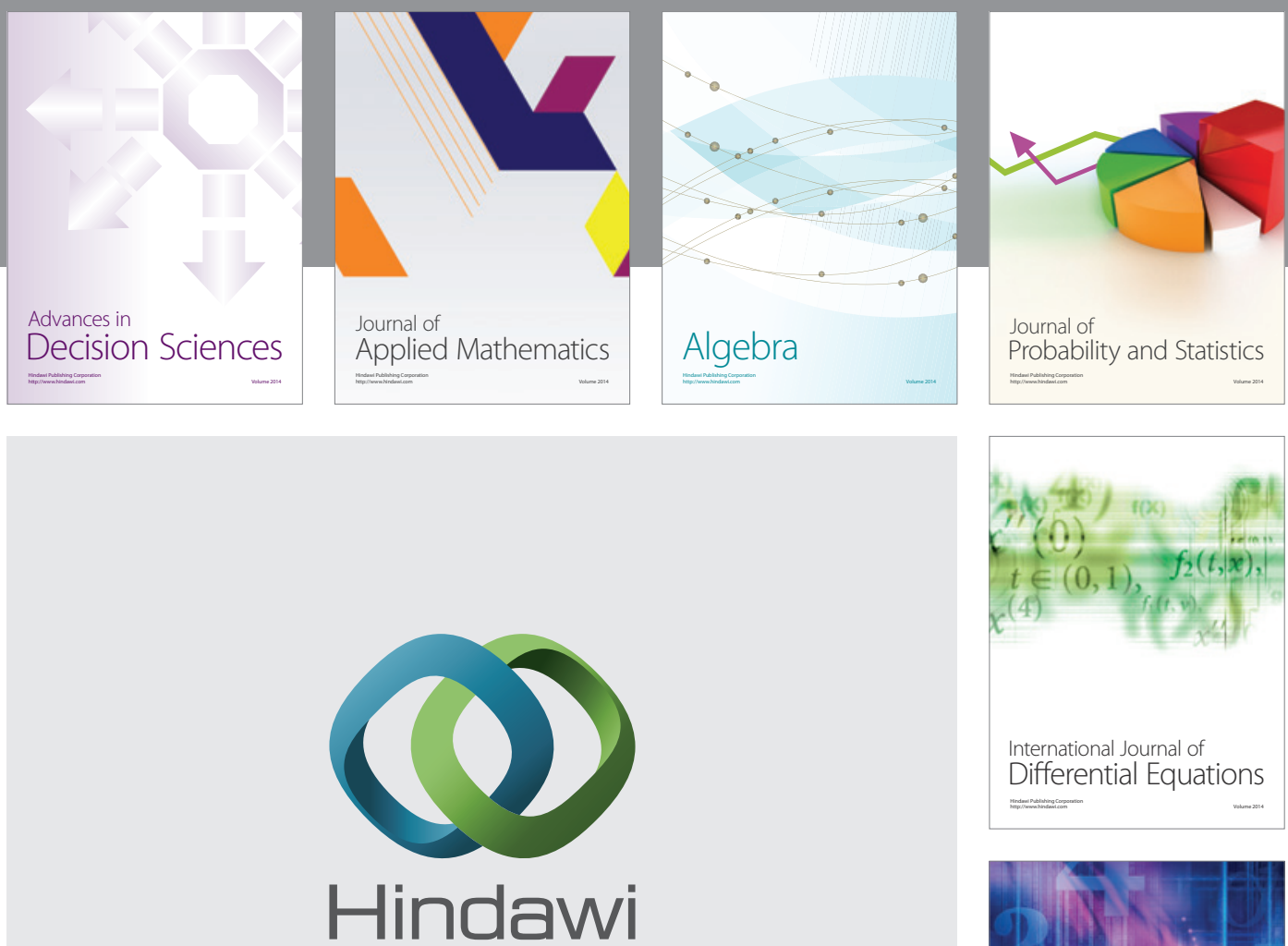

Submit your manuscripts at http://www.hindawi.com
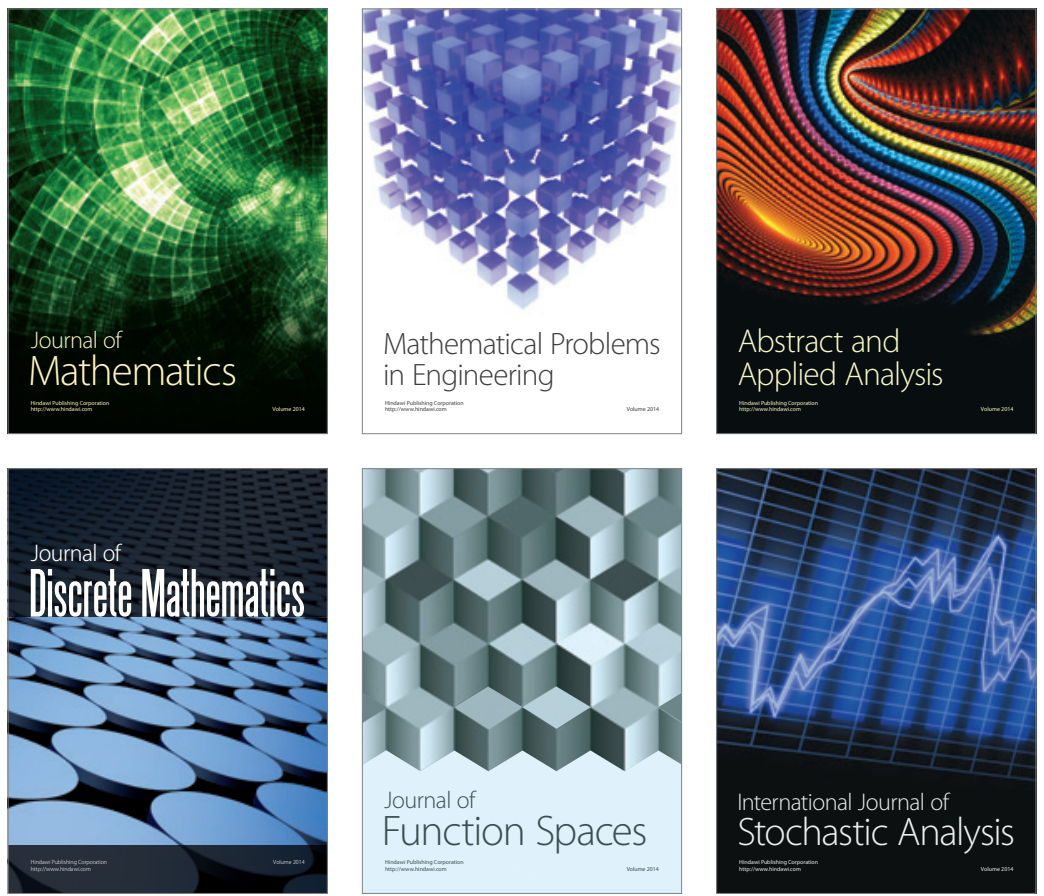

Journal of

Function Spaces

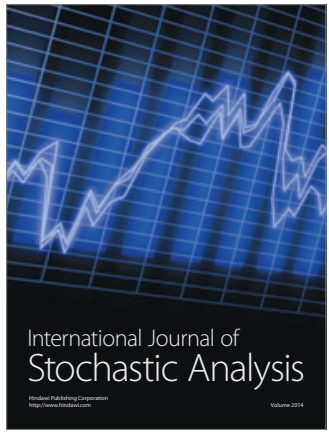

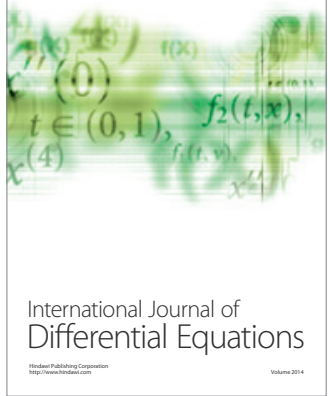
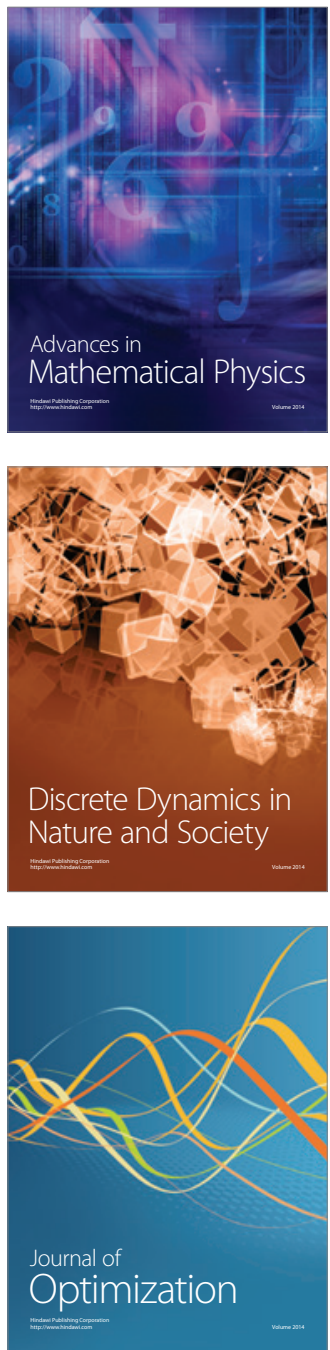